\title{
Relationship between Correcting Code and Module Technique in Hiding Secret Data*
}

\author{
Phan Trung Huy ${ }^{1}$, Nguyen Hai Thanh ${ }^{2}$, and Cheonsick Kim ${ }^{3}$ \\ ${ }^{1}$ Hanoi University of Science and Technology, Hanoi, Vietnam \\ huypt-fami@mail.hut.edu.vn, huyfr2002@yahoo.com \\ ${ }^{2}$ Ministry of Education and Training, Hanoi, Vietnam \\ nhthanh@moet.gov.vn \\ ${ }^{3}$ Dept. of Computer Engineering Sejong University, \\ 98 Gunja-Dong, Gwangjin-Gu, Seoul 143-747, Korea \\ mipsan@paran.com
}

\begin{abstract}
In this paper, we show the role of modules over rings of finite characteristics in data hiding area. Applications of correcting codes and covering functions in data hiding are shown as special cases of our module approach. Applications of modules over rings of characteristic 2 to design new embedding schemes for hiding secret data in binary images are introduced.
\end{abstract}

Keywords: module, ring, characteristic 2, data hiding, binary image, steganography, correcting codes, covering function, MSDR.

\section{Introduction}

Data hiding can be applied in copyright, annotation, and communication, and can be achieved by altering some nonessential pixels in the cover image. For example, in a given color image (including grayscale image), the least-significant bit (LSB) of each pixel can be changed to embed the secret data. However, two color images embedded by secret data are very sensitive and can be easily detected by the human eyes. One of the most challenging problems is hiding the secret data into binary images with a high ratio of secret data, and low image distortion. In case of palette images, ones need to prevent steganalysis, especially to histogram-based attacks (see for examples some analysis in [18], if the alpha ratio of the number of changed pixels to the number of total pixels of a given palette image is lower than 0.1 , it is very difficult to guess if the image contains hidden data. In block-based approaches, each binary image is partitioned into binary blocks of the same size $N$, each block can be seen as an $N$-bit string of size $N$. In such a block $F$ of size $N$, by taking WL scheme [16] one can embed one bit by changing at most one bit of $F$. From CPT scheme proposed by Chen-Pan-Tseng (2000) [3], in $F$ one can embed $r=\left\lfloor\log _{2}(N+1)\right\rfloor$ bits, by changing at most two bits of $F$.

\footnotetext{
* This work is partially supported by Vietnamese National Foundation for Science \& Technology Development (NAFOSTED).
} 
By correcting codes approach, with notion of covering codes, Crandall [5] refers to an unpublished article by Bierbrauer [1] that brings deep techniques to data hiding area, based on the point of view of a coding theorist $[10,12,13]$. The connection between linear covering codes and steganography had also appeared in one paper by Galand and Kabatiansky [6], and covering codes in [13] dating back 1994 by R. Struik. In a nice paper [7] written by J. Bierbrauer and J. Fridrich, the authors described and extended the original Bierbrauer's work[1] which show rich contributions of covering functions to data hiding area. As shown in their paper, in binary images or in binary data formed from LSB planes of three component colors Red, Green, Blue of pixels, in true color images, some schemes have reached the maximum secret data ratio $\left(\mathrm{MSDR}_{\mathrm{k}}\right)$ based on the number of secret bits which can be embedded in a block $F$ of $N$ pixels with the restrictions: in $F$, at most $k$ bits can be changed $(k=1,2,3)$. Several works $[2,4,5,6,7,9,14,15]$ introduced the powerful applications of approaches based on correcting codes in data hiding.

In this paper, we introduced an application of modules over rings of characteristic 2 to data hiding area, for binary images the main case of our interest. It can be seen that this idea will be easily extended to others characteristics for different formats on multimedia environment. The relationship between two methods in data hiding, by module and by correcting codes methods is considered. It is shown that hiding secret data based on correcting codes is the special case of hiding data by module over rings of characteristic 2 . Some new schemes for data hiding by module method are introduced, showing the advantage and flexible of module approach to data hiding area.

The paper is divided into 5 sections. Following the introduction section, section 2 recalls applications of linear correcting codes and covering codes in binary data hiding. Due to [11] we recall the notion " $k$-maximal secret data ratio" $\left(M S D R_{k}\right)$ of secret bits embedded in each block $F$ of $N$ pixels in binary images with the restriction that at most $k$-pixels can be changed in $F$. As shown, the results of covering codes which reached these limits. In section 3 we focus on modules over the ring $\mathbf{Z}_{2}$ of integers modulo 2, the main subject of this paper. Notions of $k$-base on module and $k$ embedding scheme are introduced. Some aspects of $M S D R$ are considered. To get new 2-bases of $\mathbf{Z}_{2}$-modules for new 2-embedding schemes applied to binary images, a designing method is given. In section 4 we show the relationship between correcting codes, covering function with module method, and present in details the arguments via an example of using Hamming code $(7,4)$ in data hiding. As experimental results, some 2-bases obtained by our program are presented and their applications in data hiding schemes are discussed. Conclusion is the content of section 5. The general applications of modules over the rings of characteristics $q, q>1$, specially for the special case of ring $\mathbf{Z}_{q}$ of integers modulo $q$, are discussed for future study.

\section{Error Correcting Codes and Covering Codes}

\subsection{Correcting Codes in Steganography}

As shown in the survey work [7] of J. Bierbrauer and J. Fridrich, the covering function technique is originated from error correcting codes area and this brings to 
steganography powerful ways to design high quality schemes for embedding secret data. For examples, the matrix encoding technique used in F5 algorithm by Westfeld [15] permit us to modify at most 1 pixel among $2^{k}-1$ pixels to hide $k$ secret bits. The distortion of image then is reduced with the high ratio of the embedding scheme, which reached $\mathrm{MSDR}_{1}$. Matrix encoding technique using correcting codes show that after the embedding phase, the syndromes generated by a parity check matrix in the extracting phase will present exactly secret data embedded in stego-images. In covering code technique, the covering radius of the code reflects the maximal number of pixels changed to embed secret data and the dimension of code reflects the capacity of bits can be embedded, by a scheme used this approach.

Given the linear space $W_{n}=\mathbf{F}^{n}{ }_{q}$ of dimension $n$ over the finite field $\mathbf{F}_{q}$ and a natural number $d>1$. Due to Hamming's work origined from 1947, an error correcting code over $\mathbf{F}_{q}$ is defined as a subset $C \subseteq W_{n}$ for which $d$ is the minimum distance between two distinct code words $x \neq y \in C$. In error correcting codes area, each codeword $v \in C$ can be seen as an (exact) encoded bit string we need to transmit on a noise channel. If there are at most $r=\lfloor d / 2\rfloor$ errors appear on transmission, that is $v$ is changed to $v^{\prime}=v+e$ for some error vector $e$ whose Hamming weight $w(e) \leq r$, then we can correct $v$ ' to recover $v$ after erasing $e$, by some methods detecting $e$. The size of $C$ is defined as $|C|$ - the number of code words in $C$.

If the code is linear, we can find some effective ways to detect the error vector e.

A linear code $C$ of type $[n, k, d]$ is a linear subspace of $W_{n}$ having its dimension $k<$ $n$, with $d$ the minimum (Hamming) distance between distinct code words $x \neq y \in C$. The covering radius $\rho$ of code $C$ is defined as $\rho=\max _{v} \in \mathrm{W} d(v ; C)$, where $d(v ; C)$ denotes the minimum Hamming distance from the vector $v$ to the code $C$. A parity check matrix of $C$ is a matrix $H$ of size $t \times n, t=n-k$, which permit us to obtain for any $v \in W_{n}$ its syndrome vector $s(v)=H . v^{T}$, where $v^{T}$ is the column vector form of $v$, so that $s(v)=0$ if and only if $v \in C$. The syndrome $s(v)$ is used to detect and correct the error $e$ if $w(e)=k$ (the number of errors), $k \leq r=\lfloor d / 2\rfloor$, appeared on transmission, to recover the correct message $v$ from the received message $v^{\prime}=v+e$ as follows: since $s\left(v^{\prime}\right)=H \cdot v^{\prime}=H \cdot(v+e)^{T}=H \cdot v^{T}+H \cdot e^{T}=s(v)+s(e)=s(e)$, we find $e$ so that $\mathrm{s}(e)=\mathrm{s}\left(v^{\prime}\right)$ and $w(e) \leq r=\lfloor d / 2\rfloor$, and correct $v^{\prime}$ to $v=v^{\prime}-e$. To obtain an efficient way recognizing the error $e$, the coset technique is developed.

A coset $C+v$ is the set of all vectors in $W_{n}$ with the same syndrome $s(v)$. A vector $l_{r(v)}$ of the minimum weight in $C+v$ can be called a leader of the coset. We define the syndrome map $s: W_{n} \rightarrow W_{t}$ by $s(v)=H . v^{T}$.

In steganography (see [7,9]), one can make use of the syndrome map $s: W_{n} \rightarrow W_{t}$ as the extracting map of the embedding scheme $[n, t, \rho]$ which can be defined by:

1. Let $n$ and $t$ be positive integers, $t \leq n$, and let $X$ be a finite set of symbols. An embedding scheme of type $[n, t, \rho]$ over $X$ is given by a pair of maps $c: X^{t} \times X^{n} \rightarrow X^{n}$ and $s: X^{n} \rightarrow X^{t}$ such that $s(c(p, v))=p$ for all (plaintext) $p \in X^{t}$ and $v$ (stego-block) $\in X^{n}$. Maps $c$ and $s$ are the embedding and the extracting maps, respectively. The 
covering radius of the scheme is defined as $\rho=\max \left\{d(v, c(p, v)) \mid p \in X^{t}, v \in X^{n}\right\}$, where $d$ is the Hamming distance.

2. The embedding scheme $[n t, \rho]$ allows us to hide $p$ (as a string of $t$ secret symbols) into $v$ (as an $n$-string $v$ of $n$ cover symbols), by changing at most $\rho$ of $n$ cover symbols. The following algorithm shows this idea.

Coset Algorithm. Given $p, v$

a) Compute $u=s(v)-p$,

b) Set $v^{\prime}=c(p ; v)=v-l_{u}$, where $l_{u}$ is a leader of the coset $C+u$ of all the vectors in $W_{n}$ with the same syndrome of $u$. The Hamming weigh $w\left(l_{u}\right)=j \leq \rho$ together with vector $l_{u}$ show the exact $j$ positions on which we need to change with the cover vector $v$ by equation $v^{\prime}=v-l_{u}$. So, $s\left(l_{u}\right)=u$. This provides us in the extract phase: by using (a) above, the syndrome $\mathrm{s}\left(v^{\prime}\right)=\mathrm{s}(v)-\mathrm{s}\left(l_{u}\right)=\mathrm{s}(v)-u=p$ is obtained, the exact plaintext as claimed.

Concretely (see, such as [7]), in case $X=\mathbf{F}_{2}$ (also $\mathbf{Z}_{2}$ ) for designing an embedding scheme $[n, t, \rho]$, we can use a covering function $\operatorname{COV}(\rho, n, t)$ which permit us to embed any $t$-bit string $p$ in any $n$-bit string $v$ (as a block of $n$ bits) by changing at most $\rho$ positions on $v$.

\section{Good Covering Codes}

In [7] the authors show some interest classes of cover functions:

1. $\operatorname{COV}\left(2,5 \cdot 2^{a-1}-1,2 \mathrm{a}+1\right)$ for $a \geq 1$ by equation (1).

2. $\operatorname{COV}\left(2,6 \cdot 4^{a-1}-1,4 a\right), a \geq 2$ by equation (4). The first members of this family are $\operatorname{COV}(2,23,8), \operatorname{COV}(2,95,12)$.

By our interest, for high quality of stego-images, in this paper we focus only on small values, especially for $\rho=1,2$.

\section{2 k-Maximal Secret Data Ratio of Embedded Bits}

In this part, given an image $G$, for simplicity we only concentrate on one fixed block $F=\left(F_{1}, F_{2}, . ., F_{N}\right)$ of $N$ pixels of $G$, and $F$ is considered as a vector of dimension $\left.N\right)$. In $F$ each entry $F_{i}$ can be understood as a pixel whenever the index $i$ is referring, also as the color of this pixel whenever its color value is mentioned. Suppose each color $F_{i}$ can be changed to $q-1$ new colors $F_{i}$ ' closed to $F_{i}$, by one of $q$-1 different ways. In the case of binary images, $q=2$. In the general case $q \geq 2$ for color images.

We consider here $k$-embedding schemes in which secret bits can be embedded in each block $F$ by changing at most $k$ entries, with $k$ small, $k=1,2$. Together with $F$, each new block $F$ ' after changing pixels of $F$ is called a configuration. Denote by $\mathrm{MSDR}_{k}$ the k-Maximal Secret Data Ratio which presents the largest number of embedded bits in each block $F$ of $N$ pixels by changing colors of at most $k$ pixels in $F$.

In the case $k=1$, since we change colors in at most one element in $F$, with $N$ elements, $1+(q-1) N$ ways can be taken. This means that for any 1 -embedding scheme, we can hide at most

$$
\operatorname{MSDR}_{1}=\left\lfloor\log _{2}(1+(q-1) \cdot \mathrm{C}(N, 1))\right\rfloor \text { secret bits in each block } F .
$$


In the cases $k=2$ or 3 , similarly, we have

$$
\begin{aligned}
& \mathrm{MSDR}_{2}=\left\lfloor\log _{2}\left(1+(q-1) \cdot \mathrm{C}(N, 1)+(q-1)^{2} \cdot \mathrm{C}(N, 2)\right)\right\rfloor \text { and } \\
& \left.\mathrm{MSDR}_{3}=\left\lfloor\log _{2}\left(1+(q-1) \cdot \mathrm{C}(N, 1)+(q-1)^{2} \cdot \mathrm{C}(N, 2)\right)+(q-1)^{3} \cdot \mathrm{C}(N, 3)\right)\right\rfloor .
\end{aligned}
$$

For example, in binary images, if $N=5$ then $M S D R_{2}=4$. In the case of grey images, with $N=1, q=16, M S D R_{1}=\left\lfloor\log _{2}(1+15.1)\right\rfloor=4$. That means in each pixel $F$ (a block of 1 pixel) if its color has 15 other ways to change, then any 4 secret bits can be hidden in $F$ by some appropriate change. This is the case achieved in [8].

\section{Modules over Rings of Characteristic 2 in Hiding Secret Data}

\subsection{Application of Modules in Hiding Secret Data}

Each (right) module $M$ over the ring $\mathbf{Z}_{q}$ is an additive abelian group $M$ with zero 0 together with a scalar multiplication "." to assign each couple $(m, \boldsymbol{t})$ in $M \times \mathbf{Z}_{q}$ with an element $m . t$ in $M$. Let $\mathbf{Z}_{q}=\{\mathbf{0}, \mathbf{1}, . ., q-\mathbf{1}\}$. We need some following basic properties, which will be used in the sequent:

P1) $m . \mathbf{0}=0 ; m .1=m$;

P2) $m+n=n+m$ for all $m, n$ in $M$.

P3) $m .(\boldsymbol{t}+\boldsymbol{l})=m . \boldsymbol{t}+m . \boldsymbol{l}$ for all $m$ in $\boldsymbol{M}, \boldsymbol{t}, \boldsymbol{l}$ in $\mathbf{Z}_{q}$.

Definition 1. Given a natural number $v>0$, a subset $U \subseteq M-\{0\}$, we call $U$ a $v$-base of $M$ if for any $x \in M-\{0\}, x$ can be presented by a linear combination of at most $v$ elements in $U$. That means there exist $n$ elements $u_{1}, u_{2}, . ., u_{n}$ in $U, n \leq v$, together with $\boldsymbol{t}_{\mathbf{1}}, \boldsymbol{t}_{2}, . ., \boldsymbol{t}_{\boldsymbol{n}}$ in $\mathbf{Z}_{q}$ such that $x=u_{1} \cdot \boldsymbol{t}_{\mathbf{1}}+u_{2} . \boldsymbol{t}_{\mathbf{2}}+. .+u_{n} . \boldsymbol{t}_{\boldsymbol{n}}$.

We call a k-embedding scheme any embedding scheme that permits in each block $\mathrm{F}$ of $N$ elements ones can change at most $k$ elements to hide data. In the case $v=1$, it is obvious that $U=M-\{0\}$ is the unique 1-base.

In this paper, the main of our interest are the cases $v=1,2$ for binary images (according to the characteristic $q=2$ and $M=\mathbf{Z}_{2} \times \mathbf{Z}_{2} \times . . \times \mathbf{Z}_{2}$ is the $n$-fold cartesian product of $\mathbf{Z}_{2}$, which can be seen as a (right) $\mathbf{Z}_{2}$-module. For binary image we have $q=2$. The addition in $\mathbf{Z}_{2}$ can be seen as the operation XOR (exclusive -OR) on bits, Each element $x=\left(x_{1}, x_{2}, . ., x_{n}\right)$ in $M$ can be presented as an $n$-bit string $x=x_{1} x_{2} . . x_{n}$, with operations defined as follows:

D1) $x+y=z_{1} z_{2} . . z_{n}$ where $z_{i}=x_{i} \oplus y_{i}, i=1, . ., n$, For $x=x_{1} x_{2} . . x_{n}, y=y_{1} . . y_{n}$ in $M, \boldsymbol{k}$ in $\boldsymbol{Z}_{2}$,

D2) $x . \boldsymbol{k}=z_{1} z_{2} . . z_{n}$ where $z_{i}=\mathrm{x}_{i} \cdot \boldsymbol{k}=x_{i}$ AND $\boldsymbol{k}$.

Given a binary image $G$, we set $C_{G}=\mathbf{Z}_{2}=\{\mathbf{0 , 1}\}$ as the set of two colors of $G$. The color changing function Next: $\mathbf{Z}_{2} \rightarrow \mathbf{Z}_{2}$ is given by:

(3.1) $\mathbf{c}^{\prime}=\boldsymbol{c}+\mathbf{1}=\operatorname{Next}(c)$, for all $\boldsymbol{c}$ in $\mathbf{Z}_{2}$ and changing a color $\boldsymbol{c}$ means that $\boldsymbol{c}$ is replaced by $\boldsymbol{c}^{\prime}=\operatorname{Next}(\boldsymbol{c})$.

As one can see, an 1-embedding scheme can be reduced from a 2-embedding scheme. Hence, at first we consider 2-embedding schemes. 


\subsection{Application of 2-Bases for 2-Embedding Schemes}

Let $U \subseteq M-\{0\}, U$ be a 2-base of a $\mathbf{Z}_{2}$-module $M$. Suppose $|U|=n$. Consider any binary block $F=\left(F_{1}, F_{2}, . ., F_{\mathrm{s}}\right)$ of a given binary image $G$, and binary secret key $K=$ $\left(K_{1}, K_{2}, . ., K_{s}\right), F_{i}, K_{i} \in \mathrm{Z}_{2}, i=1, . ., N$.

Suppose $s \geq n$. For $F$, we can assign a surjective function $h_{F}:\{1,2, \ldots, N\} \rightarrow U$ as a weight function of indexes $i$ of $F_{i}$. Since $F$ is fixed in the scope, for simplicity we write $h$ instead. We can embed any secret element $d \in M$ in $F$ by changing colors of at most 2 elements in $F$ as the following 2-embedding scheme.

\subsubsection{Embedding a Secret Element d}

Set $S[F, K]=\sum_{l \leq i \leq N} h(i) \cdot T_{i}$, by taking operations on the $\mathbf{Z}_{2}-$ right module $M$.

Step 0) Given a secret key as a binary vector $K=\left(k_{0}, k_{1}, . ., k_{\mathrm{N}}\right), k_{i} \in \mathbf{Z}_{2}$.

Change the color $F_{i}$ of each $F_{i} \in F$ into a marked color $T_{i}=F_{i}+k_{i}$ (in $\mathbf{Z}_{2}$ ).

We present this computation by $T=F \oplus K$;

Step 1) Compute $m=S[F, K]$;

Step 2) Compare $m$ and $d$ :

- Case $m=d$ : keep $F$ intact;

- Case $d \neq m$ : then find $d-m=a$, for some $a \in M-\{0\}$. There are two following cases happen:

i) $a \in U$ : since $h$ is surjective, there exists $F_{q}$ in $F$, such that $h(q)=a=d-m$. Then change the color $F_{q}$ to new color $F_{q}{ }^{\prime}=\operatorname{Next}\left(F_{q}\right)=F_{q}+\mathbf{1}$.

ii) $a \notin U$ : Since $U$ is a 2-base of $M$ - a $\mathbf{Z}_{2}$-module - we can find (successfully) two elements $x, y$ in $U$ such that $a=x+y$, and therefore find two entries $F_{p}, F_{q}$ in $\boldsymbol{F}$ such that $h(p)=x, h(q)=\mathrm{y}$.

Then we change $F_{p}$ to new color $F_{p}{ }^{\prime}=F_{p}+\mathbf{1}$, and change $F_{q}$ to new color $F_{q}{ }^{\prime}=F_{q}+\mathbf{1}$;

\subsubsection{Extracting the Secret Element Embedded in F}

Step 1) Computing $u=S[F, K]$;

Step 2) Return $u$ as the secret element $d$ embedded in $S$ (that is $u=d$ ).

\section{Correctness of the Method}

Theorem 1. The element $u$ extracted in step 1 of the extracting stage 3.2 .2 above is exactly the secret element $d$ hidden into $S$ in the embedding stage 3.2.1.

Proof. We need consider only the case $d \neq m$ and prove that $u=d$.

Indeed, if the step (2i) in 3.2.1 is taken place, after changing the color $F_{q}$ to $F_{q}$, $=F_{q}+\mathbf{1}$ by step 2(ii) in 3.2.1 with $h(q)=a=d-m$, we get $T_{q}{ }^{\prime}=T_{q}+\mathbf{1}$. Then,

$u=\sum_{l \leq q \neq i \leq N} h(i) \cdot T_{i}+h(q) \cdot\left(T_{q}+\mathbf{1}\right)=\sum_{l \leq q \neq i \leq N} h(i) \cdot T_{i}+h(q) .\left(T_{q}\right)+h(q) . \mathbf{1}$

$=\sum_{l \leq i \leq N} h(i) \cdot T_{i}+h(q)=m+d-m=d$. 
For the case that the step (2ii) in 3.2.1 is taken place, using $d-m=a=x+y, h(p)=x$, $h(q)=y$, by the same arguments we deduce $T_{p}{ }^{\prime}=T_{p}+\mathbf{1}$ and $T_{q}{ }^{\prime}=T_{q}+\mathbf{1}$. Therefore by properties of modules

$$
\begin{aligned}
u & =\sum_{1 \leq p, q \neq i \leq N} h(i) \cdot T_{i}+h(p) \cdot T_{p}{ }^{\prime}+h(q) \cdot T_{q}=\sum_{1 \leq p, q \neq i \leq N} h(i) \cdot T_{i}+h(p) \cdot T_{p}+h(p)+h(q) \cdot T_{q}+h(q) \\
& =\sum_{l \leq i \leq N} h(i) \cdot T_{i}+h(p) \cdot T_{p}+h(q) \cdot T_{q}+h(p)+h(q) \\
& =\sum_{l \leq i \leq N} h(i) \cdot T_{i}+\mathrm{h}(p)+\mathrm{h}(q)=m+a=m+d-m=d . \text { This completes the proof. } \|
\end{aligned}
$$

Example 1. The subset $U=\{0001,0010,0100,1000,1111\}$ is a 2-base of the module

$M=\mathbf{Z}_{2} \times \mathbf{Z}_{2} \times \mathbf{Z}_{2} \times \mathbf{Z}_{2}$. Therefore, we can use it to hide data. In any block $F$ of 5 pixels, we can change at most two pixels to hide 4 bits. That is the $M S D R_{2}$ is obtained: $\operatorname{MSDR}_{2}=\left\lfloor\log _{2}(1+5(5+1) / 2)\right\rfloor=4=\left\lfloor\log _{2}(|M|)\right\rfloor$.

Remark 1. Applications of $k$-bases for $k$-embedding schemes are similarly established, for any $k>0$, hence we do not mention in details.

\subsection{Designing 2 - Bases of $\mathrm{Z}_{2}{ }^{\mathrm{n}}$}

In this part we introduce a method to design 2-bases of $\mathbf{Z}_{2}{ }^{n}$ inductively.

Denote by $V_{n}=\mathbf{Z}_{2}{ }^{n}=\mathbf{Z}_{2} \times \mathbf{Z}_{2} \times$.. $\times \mathbf{Z}_{2}$ the $\mathbf{Z}_{2}$ - module whose elements can be presented simply as the form $b=b_{n} b_{n-1} . . b_{1}$, an $n$ - bit strings.

Denote by $P R_{k}\left(V_{\mathrm{n}}\right)$ the projection getting $k$ right components in $V_{n}$ and $P L_{k}\left(V_{n}\right)$ the projection getting $k$ left components in $V_{n}$.

Concretely, $P R_{k}\left(b_{n} b_{n-1} . . b_{r} . b_{1}\right)=b_{k} b_{k-1} . . b_{1}$ and $P L_{k}\left(b_{n} b_{n-1} . . b_{2} b_{1}\right)=b_{n} b_{n-1} . . b_{n-k+2} b_{n-k+1}$.

Denote by $C L_{k, t}$ a class of 2-bases $X$ of $V_{n}$ satisfying $|X|=2^{k}+2^{t}-3, n=k+t$, and $P L_{k}(X)=V_{k}$.

Denote by $C R_{k, t}$ a class of 2-bases $X$ of $V_{n}$ satisfying $|X|=2^{k}+2^{t}-3, n=k+t$, and $P R_{t}(X)=V_{t}$.

Lemma 2. If $X$ is a 2-base of $V_{n}$ such that $P L_{m}(X)=V_{m}$ for some integer $0<m<n$, then there exist a 2-base $Y$ of $V_{n+1}$ such that $P L_{m}(Y)=V_{m}$ and $|Y|=|X|+2^{r}, m+r=n$.

Proof. Define $Y$ is the set of all $n+1$-bit string $x$ in one of two following forms:

(i) $b_{n} b_{n-1} . . b_{1} 0$, with any $b_{n} b_{n-1} . . b_{1}$ in $X$ and whose right most bit is 0 .

(ii) $00 . .0 x_{r} x_{r-1} . . x_{1} 1$ with any $x_{r} x_{r-1} . . x_{1} \in V_{r}$.

By assumption on $X$, any element of the form $y_{1} . . y_{m} 0$ in $V_{n+1}$ can be presented as a linear combination of at most two elements in $Y$. For any element of the form $y_{1} . . y_{m} x_{1} . . x_{r} 1$ in $V_{n+1}$, by $P L_{m}(X)=V_{m}$ there exists n-bit string of $X$ of the form $y_{1} . . y_{\mathrm{m}} v_{1} . . v_{r}$, for some $r$-bit string $v_{1} . . v_{\mathrm{r}}$. So $y_{1} . . y_{m} v_{1} . . v_{r} 0$ belongs to $Y$ by definition.

Define $u_{1} . . u_{\mathrm{r}}=x_{1} . . x_{r} \oplus v_{1} . . v_{r}$. Then $y_{1} . . y_{m} x_{1} . . x_{r} 1=00 . .0 u_{1} . . u_{r} 1 \oplus y_{1} . . y_{1} v_{1} . . v_{r}$. Hence $Y$ is a 2-base of $V_{n+1}$. Obviously, $|Y|=|X|+2^{r}, m+r=n$ and $P L_{m}(Y)=V_{m}$. $\|$

Remark 2. By duality, from a 2-base $X$ satisfying $P R_{r}(X)=V_{r}$, we can define a 2-base $\mathbf{Z}$ of $V_{n+1}$ having all elements in one of two forms: $1 y_{1} . . y_{\mathrm{m}} 00 . .0$, with any $m$-bit string $\mathrm{y}_{1} . . \mathrm{y}_{\mathrm{m}}$ in $V_{m}$, and $0 x_{1} . . x_{m} u_{1} . . u_{r}$, with any $n$-bit string $x_{1} . . x_{m} u_{1} . . u_{\mathrm{r}}$ in $X$. 
Theorem 3. For any $n \geq 4$, there exist 2-bases $X$ in $C L_{m, r}, Y$ in $C R_{m, r}$ such that if $m, r$ $>1, m+r=n$ then $|X|,|Y| \leq 2^{m}+2^{r}-3$.

Proof. Firstly, one can see that:

a) $\mathrm{CL}_{2,2}$ contains the set $Z=\{0001,0010,0100,1110,1001\}$, this set satisfies the claim $|Z|=5=2^{2}+2^{2}-3$, and $C_{2,2}$ contains the set $T=\{1000,0100,0010,0111,1001\}$ with $|T|=5$ which satisfies the claim.

b) For all $\mathrm{CL}_{m, r}, \mathrm{CR}_{m, r}, m+r=n, n \geq 4, m, r>1$, one can prove easily the theorem by induction on $n$, starting from two 2-bases $Z, T$ above and applying Lemma 2 together with Remark 2 by duality.

\section{Example 1}

a) the class $\mathrm{CL}_{2,1}$ contains the set $X=\{110,100,010,001\}$, and $\mathrm{CR}_{1,2}$ contains the set $Y$ $=\{011,001,010,100\}$.

Generally, for any $n>0$, we can define the set $X$ contains all $n+1$ - bit strings in one of two forms: $b_{n} b_{n-1} . . b_{1} 0 \neq 00 . .0$ and $00 . .01$. Then $X$ belongs to $\mathrm{CL}_{n, 1}$. We define the set $Y$ contains all $n+1$ - bit strings of two forms: $0 b_{n} b_{n-1} . . b_{1} \neq 00 . .0$ and $100 . .0$, then $Y$ belongs to $\mathrm{CL}_{n, 1}$.

b) $\mathrm{CL}_{2,3}$ contains the set of 9 elements $X=\{00010,00100,01000,11100,10010$, 00001, 00011, 00101, 00111\}

c) $\mathrm{CL}_{3,3}$ contains the set of 13 elements $\{000010,000100,001000,011100$, 010010, 000001, 000011, 000101, 000111, 100000, 110000, 101000, 111000\}.

\section{Correcting Codes and Module Method in Data Hiding}

\subsection{Correcting Code and Covering Function as Special Cases of Module Method}

In data hiding area, by the essential relation between correcting code and covering function, for simplicity we need only to show the relation between correcting codes and module methods. Indeed, by the coset algorithm in the part 2.1 , section 2 , in the extract phase, a plaintext $p$ hidden in the cover-string $v^{\prime}$ can be extracted from $v$ ' by computing the syndrome of $v^{\prime}$ as $p=\mathrm{s}\left(v^{\prime}\right)=H . v^{\prime}$, where $v^{\prime}=v-l_{u}$ and $H$ is the parity checking matrix of size $t \times n$ which can be presented as $H=\left(C_{1}, \ldots, C_{\mathrm{i}}, . ., C_{\mathrm{n}}\right)$, where each column vector $C_{i}$ with size $t \times 1$ is considered an element in $\mathrm{W}_{t}$, a $\mathbf{Z}_{2}$ - module. Suppose $v^{\prime}=\left(x_{1}, . ., x_{n}\right), x_{i} \in \mathbf{Z}_{2}, i=1, \ldots, n$. It is obvious that the syndrome $s\left(v^{\prime}\right)=H . v^{\prime}=$ $\Sigma_{1 \leq i \leq n} C_{i} \cdot x_{i}$ is exactly the sum we compute by module method, where the weight function $h:\{1, . ., \mathrm{n}\} \rightarrow M-\{0\}$ is defined by $h(i)=C_{i}$ for all $i=1, . ., n$ with the $\mathbf{Z}_{2^{-}}$ module $M=\mathrm{W}_{t}$. Computing $v^{\prime}=v-l_{u}$ means that we need to find some positions on $v$ to flip if in the coset leader $l_{u}$ of $u=s(v)-p$, the corresponding positions are different from 0 . In the following example, we present the arguments in details.

Example 2. The Hamming code can supply us an instance for module approach in hiding data. In details, we have the embedding scheme [7,3,1] by using the Hamming code $(7,4)$, taking $W_{3}-\{0\}=\{1,2, . ., 7\}$ and considering each column $C_{1}, C_{2}, . ., C_{7}$ of the parity checking matrix $H$ of size $3 \times 7$ as a 3-bit presentation of these numbers 


$$
\begin{aligned}
& H=\left(\begin{array}{lllllll}
1 & 0 & 1 & 0 & 1 & 0 & 1 \\
0 & 1 & 1 & 0 & 0 & 1 & 1 \\
0 & 0 & 0 & 1 & 1 & 0 & 1
\end{array}\right) \\
& \begin{array}{lllllll}
C_{1} & C_{2} & C_{3} & C_{4} & C_{5} & C_{6} & C_{7}
\end{array}
\end{aligned}
$$

Fig. 1. Parity checking matrix $H$ in Hamming code $(7,4)$

Each block $F$ of the binary image can be seen as a column vector $u$ of 7 entries: $u=$ $\left(x_{1}, x_{2}, \ldots x_{7}\right)^{\mathrm{T}}$. Then taking operations on $\boldsymbol{Z}_{2}$-module we can write $H . u=C_{1} \cdot x_{1}+C_{2} \cdot x_{2}+$ $\ldots+C_{7} . x_{7}$ where each column $C_{i}$ can be seen as a vector in $V_{3}=Z_{2}^{3}$ and the set $C=\left\{C_{1}\right.$, $\left.C_{2}, C_{3}, C_{4}, C_{5}, C_{6}, C_{7}\right\}$ is nothing but an 1-base in $\mathbf{Z}_{2}$ - module $V_{3}$. For security reason, one can choose an extra binary key $k$ - as a column vector of 7 entries, $k=\left(k_{1}, k_{2}, . ., k_{7}\right)^{\mathrm{T}}$ and taking operation XOR, with $u$ we get $v=u \oplus k=\left(y_{1}, y_{2}, . ., y_{7}\right)$ and $H . v=C_{1} \cdot y_{1}+C_{2} \cdot y_{2}+. .+C_{7} \cdot y_{7}$.

Replacing a position $x_{j}$ in $u$ by $x_{j} \oplus 1$ implies that the same position $y_{j}$ in $v$ is replaced by $y_{j} \oplus 1$. This gives us the marked vector $v^{\prime}$ satisfying the equation $H . v^{\prime}=$ $H . v \oplus C_{j}$. Now, suppose $H . v=$ e and we need to hide a vector $d$ (of 3 bits) which is considered as an element in $W_{3}$. We can flip at most one position $x_{j}$ in $u$ to hide $d$ as follows:

Case $d=e$, the block $u$ is kept intact, so that in the extracting phase, ones recover H.v $=$ $d$. Case $d \neq e$, or equivalent, $e-d \neq 0$, we can find in C (an 1-base of $W_{3}$ ) a vector $C_{j}$ so that $C_{j}=e-d$ (that means $C=e \oplus d$ in $W_{3}$ ). After flip $x_{j}$ to $x_{j} \oplus 1$ in $u$, we have $H . v^{\prime}=$ $H . v \oplus C_{j}=e \oplus e \oplus d=d$, the result one needs to recover in the extract phase. Let us remark that the sum $C_{1} \cdot y_{1}+C_{2} \cdot y_{2}+. .+C_{7} \cdot y_{7}$ is exactly the result we get by the steps in 3.2.1 where $C_{i} \cdot y_{i}$ is nothing but $\mathrm{h}(i) . T_{i}$ in that steps, with $h(i)=C_{i}$ for $i=1,2, \ldots, 7$.

\subsection{Experimental Results for Finding 2-Bases}

As some results generated from our program, we obtained:

(i) The 2-base $X=\{0001,0010,0100,1110,1001\}$ and $Y=\{1000,0100,0010$, $0111,1001\}$ provide us 2- embedding schemes which permit in each block of 5 pixels, by changing at most two pixels one can hide 4 bits. Hence regarding 2embedding schemes, our schemes can be seen as some expanded for the list $\operatorname{COV}\left(2,6 \cdot 4^{a-1}-1,4 a\right)$ with $a=1$, by equation (4)[7] mentioned in section 2 .

(ii) The 2-base $X=\{1,2,3,4,5,6,8,16,24,37,45,53,58\}$ and $Y=\{1,2,3,4,5$, $6,8,16,24,32,47,55,63\}$ in $W_{6}=\mathbf{Z}_{2}{ }^{6}$ (the numbers can be seen as 6-bit strings). We provide new 2-bases with 13 elements, which permit us to hide 6 bits in each block of 13 pixels. Then we can extend them by the techniques mentioned in Lemma 2 to obtain new 2-bases for hiding 7-bit strings.

\section{Conclusion}

By flexibility of module approach for which correcting codes and covering function techniques are considered as the special cases, we can offer more new and powerful 
schemes to hide data without of using radius or distances as in correcting and covering codes.

In color images (24bpp with three channels Red, Green, Blue), or in palette images, especially for grayscale images, ones can obtain a higher ratio of secret bits hiding in each block of images by using some other module, such as $\mathbf{Z}_{q}, q>2$.

Several works consider some ways to hide as much as secret data in each block of pixels with low image distortion if it is possible. In preventing from steganalysis attacks, ones need a very high quality of stego-images, generally in palette images, hiding bits in each pixel is not good enough for security reason. In these situations, changing only a small $k=1,2,3$ pixels in each block of pixels, using a huge number of key matrices to prevent effectively from exhausted attacks, by some k- embedding scheme modified for color images we can obtain stego-images with the high quality. These will be studied in future works.

\section{References}

1. Bierbrauer, J.: Crandall's problem (1998) (unpublished), http: / / www.ws.binghamton. edu/fridrich/covcodes.pdf

2. Chang, C.C., Kieu, T.D., Chou, Y.C.: A High Payload Steganographic Scheme based on $(7,4)$ Hamming Code for Digital Images. In: Electronic Commerce and Security 2008 Symposium, pp. 16-21 (2008)

3. Chen, Y., Pan, H., Tseng, Y.: A secure of data hiding scheme for two-color images. In: IEEE Symposium on Computers and Communications (2000)

4. Kim, C., Shin, D., Shin, D.: Data Hiding in a Halftone Image Using Hamming Code (15, 11). In: Nguyen, N.T., Kim, C.-G., Janiak, A. (eds.) ACIIDS 2011, Part II. LNCS, vol. 6592, pp. 372-381. Springer, Heidelberg (2011)

5. Crandall, R.: Some notes on steganography. Posted on steganography mailing list (1998), http://os.inf.tu-dresden.de/_westfeld/crandall.pdf

6. Galand, Kabatiansky, G.: Information hiding by coverings. In: Proceedings of the IEEE Information Theory Workshop, pp. 151-154 (2004)

7. Bierbrauer, J., Fridrich, J.: Constructing Good Covering Codes for Applications in Steganography. In: Shi, Y.Q. (ed.) Transactions on DHMS III. LNCS, vol. 4920, pp. 1-22. Springer, Heidelberg (2008)

8. Lee, C.F., Chen, H.L.: A novel data hiding scheme based on modulus function. The Journal of Systems and Software 83, 832-843 (2010)

9. Ould Medeni, M.B., Souidi, E.M.: A Novel Steganographic Protocol from Error-correcting Codes. Journal of Information Hiding and Multimedia Signal Processing 1(4) (October 2010) ISSN 2073-4212

10. Nordstrom, A.W., Robinson, J.P.: An optimum nonlinear code. Information and Control 11, 613-616 (1967)

11. Phan, T.H., Nguyen, H.T.: On the Maximality of Secret Data Ratio in CPTE Schemes. In: Nguyen, N.T., Kim, C.-G., Janiak, A. (eds.) ACIIDS 2011, Part I. LNCS (LNAI), vol. 6591, pp. 88-99. Springer, Heidelberg (2011)

12. Preparata, F.P.: A class of optimum nonlinear double-error-correcting codes. Information and Control 13, 378-400 (1968)

13. Struik, R.: Covering Codes. Ph.D. dissertation, Eindhoven (1994) 
14. Zhang, W., Zhang, X., Wang, S.: Maximizing Steganographic Embedding Efficiency by Combining Hamming Codes and Wet Paper Codes. In: Solanki, K., Sullivan, K., Madhow, U. (eds.) IH 2008. LNCS, vol. 5284, pp. 60-71. Springer, Heidelberg (2008)

15. Westfeld, A.: F5-A Steganographic Algorithm High Capacity Despite Better Steganalysis. In: Moskowitz, I.S. (ed.) IH 2001. LNCS, vol. 2137, pp. 289-302. Springer, Heidelberg (2001)

16. Wu, M.Y., Lee, J.H.: Anovel data embedding method for two-color fascimile images. In: Proceedings of International Symposium on Multimedia Information Processing. ChungLi, Taiwan, R.O.C (1998)

17. Zhang, X., Wang, S.: Analysis of Parity Assignment Steganography in Palette Images. In: Khosla, R., Howlett, R.J., Jain, L.C. (eds.) KES 2005. LNCS (LNAI), vol. 3683, pp. 1025-1031. Springer, Heidelberg (2005)

18. Zhang, X., Wang, S.: Vulnerability of pixel-value differencing steganography to histogram analysis and modification for enhanced security. Pattern Recognition Letters 25, 331-339 (2004) 